\title{
Comprendiendo el afrontamiento y la calidad de vida en adolescentes con espina bífida
}

\author{
Andrea Salinas-Rosas ${ }^{1}$ \\ Richard Paucar-Yangua ${ }^{2}$
}

RESUMEN: Objetivo: determinar la fuerza de asociación entre los estilos y estrategias de afrontamiento y la calidad de vida en adolescentes con espina bífida (EB). Metodología: el estudio fue de corte transversal y de tipo explicativo. El tamaño de la muestra fue de 70 adolescentes con EB de ambos sexos y edades comprendidas entre 12 y 18 años. Las variables de estudio fueron la calidad de vida y el afrontamiento. Asimismo, se incluyeron variables sociodemográficas. Se empleó el modelo de regresión logística ordinal donde se calcularon Odds Ratios (OR). Resultados: el $25.7 \%$ de los encuestados reportó una muy buena calidad de vida y el 18.7 presentó un estilo de afrontamiento de resolver el problema. En cuanto al estilo resolver el problema (OR: 1.06) y la estrategia de buscar diversiones relajantes (OR: 1.28) se asociaron con niveles altos de la calidad de vida. Sin embargo, el afrontamiento no productivo (OR: 0.96) y las estrategias de la falta de afrontamiento (OR: 0.75), auto inculparse y reservarlo para sí (OR: 0.81) se asociaron a una disminución de la calidad de vida. Conclusiones: los niveles altos de la calidad de vida se asocian fuertemente con el estilo resolver el problema. Sin embargo, una disminución de la calidad de vida se asocia con el estilo afrontamiento no productivo y las estrategias de la falta de afrontamiento y auto inculparse. Por ello, es necesario crear programas de intervención para reducir las secuelas de estilos y estrategias de afrontamiento no productivos en los adolescentes con EB.

PALABRAS CLAVE:

Afrontamiento; Calidad de vida; Espina bífida.

Citar como:

Salinas A, Paucar R. Comprendiendo el afrontamiento y la calidad de vida en adolescentes con espina bífida. CASUS. 2017;2(3):139-146. 


\section{INTRODUCCIÓN}

La espina bífida (EB) es una malformación congénita debida a la carencia de ácido fólico en la etapa embrionaria durante la cuarta semana de gestación, entre 25 a 29 días $(1,2)$. Dicha condición hace que el tubo neural no llegue a cerrarse por completo, produciendo un quiste medular en el lugar de la lesión, teniendo secuelas permanentes a lo largo de la vida (3). En el Perú durante el 2013 la incidencia de espina bífida fue 8,3/10,000 en recién nacidos (4).

Los adolescentes con EB perciben una autoimagen peyorativa a comparación de la población general, donde su interacción social está relacionada estrechamente con el auto concepto. En este fenómeno un factor importante es la relación con sus padres (5). Por otro lado, la autoestima y autoconcepto está estrechamente relacionada con la percepción del colegio, ya que esta es una fuente de agresiones, rechazos, burlas o de aislamiento social. En este contexto, por el contrario, los adolescentes que no presentan estos problemas denotan una mayor autoestima, superiores valores felicidad general y niveles de ansiedad bajos (6)

En la adolescencia se presentan situaciones generan una capacidad de afrontamiento, ya sea con la utilización de estrategias cognitivas y/o conductuales, con el fin de adaptarse a su entorno. En esta etapa el sujeto utiliza dichas estrategias constituyendo más adelante sus propios estilos de afrontamiento (7). Por otro lado, las personas con una discapacidad vinculada a la EB afrontan eventos de la vida cotidiana con limitaciones vinculadas a la movilidad. En relación a lo anterior, se conoce que la estrategia centrada en la emoción es las más utilizada por los individuos con discapacidad motora (8).

En la adolescencia es complejo definir la calidad de vida ya que implica considerar en la etapa de vida sus aspectos biológicos, psicológicos y sociales interactuando entre sí y con el entorno. Son numerosos los factores que favorecen o desfavorecen una adecuada calidad de vida en el adolescente. Estos contribuirán en gran medida a que la persona desee salir adelante por sí misma y a buscar mayores niveles de independencia. No obstante, las creencias sociales y las de sus propias familias, las limitaciones físicas, el nivel de la lesión medular, o el usar silla de ruedas no son factores significativos para que estas personas tengan una buena o deficiente calidad de vida (9). Sin embargo, la calidad de vida en personas con EB dependerá mucho de diversos factores como los hábitos de su vida diaria, las expectativas de vida que tienen de sí mismos, las metas, sueños y objetivos que desean lograr a lo largo de su vida (9).

Las conductas y cogniciones que los individuos manejan ante una situación además del uso de los recursos personales, permiten la adaptación y transición de manera efectiva ante los problemas (10). Análogamente, la percepción en el contexto cultural, los valores, metas, objetivos, expectativas y preocupaciones determinan la satisfacción del sujeto en la vida (11). Es por ello que estas variables son relevantes en la etapa propia de la adolescencia. En una condición como la provocada por la EB conllevará a que estos procesos tengan mayor impacto en la calidad de vida de estas personas.

Teniendo en cuenta lo anterior, no se ha profundizado en cómo los estilos y estrategias de afrontamiento impactan en la calidad de vida en adolescentes con EB. Es necesario conocer con mayor precisión el comportamiento de estas variables en la población peruana para poder plantear estrategias de evaluación, intervención y seguimiento a través de programas de promoción de la salud. De esta manera se propuso como objetivo determinar la relación de los estilos y estrategias de afrontamiento y la calidad de vida en adolescentes con EB.

\section{MATERIALES Y MÉTODOS}

El estudio fue de tipo explicativo. Participaron 70 adolescentes con EB de ambos sexos con edades comprendidas entre 12 y 18 años. Se excluyeron aquellos adolescentes con discapacidad cognitiva. El muestreo utilizado fue no aleatorio. La variable dependiente fue la calidad de vida que considera a la persona en relación al medio que la rodea, de acuerdo a sus necesidades y demandas (12). Variable categórica que se midió a través del Cuestionario de Calidad de Vida relacionada a la Salud KIDSCREEN - 52. Este instrumento se basa en el modelo teórico Biopsicosocial de Engel (13). Fue categorizada en muy mala $(<184)$, mala $(184-207)$, buena $(208-222)$ y muy buena $(>222)$.

La variable afrontamiento hace referencia a un conjunto de acciones cognitivas y afectivas que surgen en respuesta a una preocupación en particular (14). Variable numérica que para su medición fue 
utilizada la Escala de Afrontamiento (ACS). Este instrumento se apoya en el modelo teórico transaccional de Lazarus y Folkman (15). El cual se divide en tres estilos (resolver el problema, afrontamiento no productivo y referido a otros) y en 18 estrategias.

Respecto a las variables sociodemográficas fueron consideradas: edad, variable categórica (12-13, 1415 y 16-18 años). Sexo, variable categórica (masculino, femenino). Grado de instrucción, variable categórica $\left(5^{\circ}\right.$ primaria, $6^{\circ}$ primaria, $1^{\circ}$ secundaria, $2^{\circ}$ secundaria, $3^{\circ}$ secundaria, $4^{\circ}$ secundaria, $5^{\circ}$ secundaria y superior). Tipos de familia, variable categórica (nuclear, monoparental, extensa y reconstituida). Diagnóstico, variable categórica (Mielomeningocele abierta, Espina bífida oculta y Mielomeningocele cerrado). Ubicación de la lesión, variable categórica (Lumbar, Sacro, Tórax, Lumbro-sacro, Dorso lumbar y Dorsal) y Ayuda para la marcha, variable categórica (camina sin ayuda, soportes, uso de silla de ruedas).

Para el análisis estadístico se utilizó el paquete estadístico STATA SE versión 12. Para el análisis descriptivo se calcularon frecuencias y porcentajes de las variables categóricas y medias para las numéricas. Se verificó la distribución de la normalidad mediante la prueba de normalidad Shapiro Wilk obteniendo como resultado una distribución normal, por lo que se procedió a utilizar la prueba paramétrica de Anova. Asimismo se empleó el modelo de regresión logística ordinal, donde se calcularon Odss Ratios (OR) e intervalos de confianza al 95\% con un p-value menor o igual a 0.05 .

Se determinó la confiabilidad del cuestionario KIDSCREEN - 52 que obtuvo un coeficiente de Alfa de Cronbach de 0.93, mientras que en sus dimensiones este coeficiente osciló entre 0.76 a 0.90. La validez del instrumento se determinó por juicio de expertos alcanzando un coeficiente $\mathrm{V}$ Aiken de 0.95 para la escala general y en sus dimensiones por encima de 0.90. Igualmente se determinó la confiabilidad de la ACS con un coeficiente de Alfa de Cronbach de 0.90. En relación al ítem - test se obtuvo un Alfa de Cronbach entre 0.89 y 0.90 .

La investigación fue aprobada por un Comité de
Ética. Con la finalidad de proteger a los participantes se obtuvo el consentimiento informado tanto de los padres de familia como de los adolescentes. Se garantizó el carácter anónimo de la información recolectada.

\section{RESULTADOS}

En la tabla 1 se muestran las características de la población de estudio. El promedio de edad fue de 15 años, asimismo las mujeres representaron el 52.9\%, prevaleciendo el grado de instrucción secundaria (84.3\%). En relación al diagnóstico se observó que el más prevalente fue la MMC abierta (72.9\%), la ubicación lumbar de la lesión fue la más frecuente (38.6\%). Del mismo modo, el uso de sillas de ruedas fue la ayuda más empleada para la marcha (50\%). En cuanto a la calidad de vida el $25.7 \%$ de los encuestados reportó una muy buena calidad de vida y el $18,7 \%$ presentó el estilo de afrontamiento de resolver el problema.

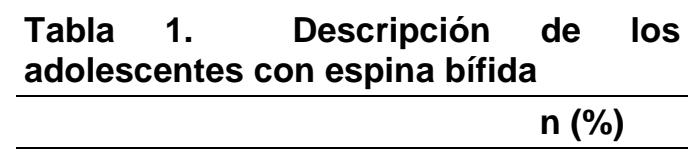

Edad m(ds)
Sexo

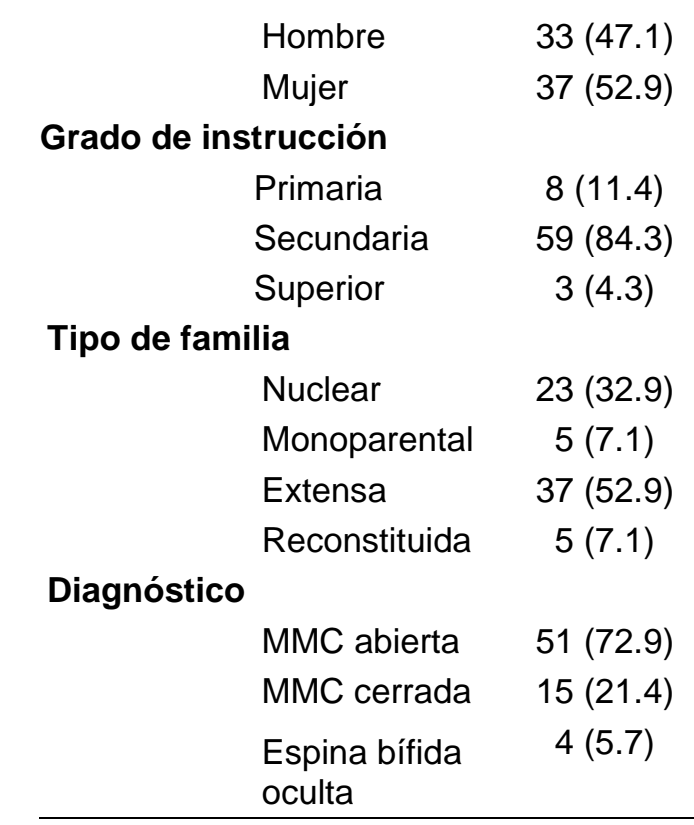


Tabla 1. continúa

\begin{tabular}{|c|c|}
\hline & n (\%) \\
\hline \multicolumn{2}{|l|}{ Ubicación de la lesión } \\
\hline Lumbar & $27(38.6)$ \\
\hline Sacro & $17(24.3)$ \\
\hline Lumbro-sacro & $15(21.4)$ \\
\hline Dorso lumbar & $2(2.9)$ \\
\hline Dorsal & $7(10.0)$ \\
\hline Tórax & $2(2.9)$ \\
\hline \multicolumn{2}{|l|}{ Ayuda para la marcha } \\
\hline $\begin{array}{l}\text { Camina sin } \\
\text { ayuda }\end{array}$ & $13(18.6)$ \\
\hline Soportes & $22(31.4)$ \\
\hline $\begin{array}{l}\text { Uso de sillas } \\
\text { de ruedas }\end{array}$ & $35(50.0)$ \\
\hline \multicolumn{2}{|l|}{ Calidad de vida } \\
\hline Muy mala & $17(24.3)$ \\
\hline Mala & $18(25.7)$ \\
\hline Buena & $17(24.3)$ \\
\hline Muy buena & $18(25.7)$ \\
\hline \multicolumn{2}{|l|}{$\begin{array}{l}\text { Estilos de afrontamiento } \\
\mathrm{m}(\mathrm{ds})\end{array}$} \\
\hline $\begin{array}{l}\text { Resolver el } \\
\text { problema }\end{array}$ & $102.8(18.7)$ \\
\hline $\begin{array}{l}\text { Afrontamiento } \\
\text { no productivo }\end{array}$ & $85.2(15.1)$ \\
\hline $\begin{array}{l}\text { Referido a } \\
\text { otros }\end{array}$ & $50.1(13.2)$ \\
\hline
\end{tabular}

Existe una asociación significativa entre el estilo de afrontamiento de resolver el problema y una muy buena calidad de vida $(\mathrm{p}=0.0001)$. Ser hombre (33.3\%), tener un nivel de estudio superior $(66.7 \%)$, provenir de una familia nuclear $(30.4 \%)$, tener el diagnóstico de MMC cerrada (26.7\%), con la ubicación de la lesión en el tórax $(50 \%)$ y caminar sin ayuda (30.8\%) presentaron una de calidad de vida muy buena, sin embargo estos resultados no fueron significativos. El resto de los indicadores se pueden observar en la tabla 2 .

En la tabla 3 se evidencia que el estilo resolver el problema se asocia con niveles altos de la calidad de vida (OR: 1.06). Sin embargo, el afrontamiento no productivo se asocia a una disminución de la calidad
Tabla 2. Asociación entre los estilos de afrontamiento y calidad de vida

\begin{tabular}{|c|c|c|c|c|c|}
\hline & \multicolumn{5}{|c|}{ Calidad de vida } \\
\hline & $\begin{array}{c}\text { Muy } \\
\text { mala } \\
(\%)\end{array}$ & $\begin{array}{c}\text { Mala } \\
(\%)\end{array}$ & $\begin{array}{c}\text { Buena } \\
(\%)\end{array}$ & $\begin{array}{c}\text { Muy } \\
\text { buena } \\
(\%)\end{array}$ & $\mathbf{p}$ \\
\hline $\begin{array}{l}\text { Estilos de } \\
\text { afrontamiento } \mathrm{m}(\mathrm{ds})\end{array}$ & & & & & 0.02 \\
\hline $\begin{array}{l}\text { Resolver el } \\
\text { problema }\end{array}$ & 88.8 & 99.8 & 106.8 & 115.5 & 0.00 \\
\hline $\begin{array}{l}\text { Afrontamiento } \\
\text { no productivo }\end{array}$ & 93.8 & 84.8 & 83.4 & 79.3 & 0.03 \\
\hline Referido a otros & 46.1 & 49.0 & 48.9 & 56.2 & 0.12 \\
\hline Edad & 15.6 & 14.9 & 15.3 & 14.7 & 0.40 \\
\hline Sexo & & & & & \\
\hline Hombre & 15.1 & 30.3 & 21.2 & 33.3 & 0.23 \\
\hline Mujer & 32.4 & 21.6 & 27.0 & 18.9 & \\
\hline Grado de instrucción & & & & & \\
\hline Primaria & 25.0 & 25.0 & 37.5 & 12.5 & 0.57 \\
\hline Secundaria & 23.7 & 27.1 & 23.7 & 25.4 & \\
\hline Superior & 33.3 & - & - & 66.7 & \\
\hline Tipo de familia & & & & & \\
\hline Nuclear & 26.1 & 17.4 & 26.1 & 30.4 & 0.76 \\
\hline Monoparental & 40.0 & - & 40.0 & 20.0 & \\
\hline Extensa & 21.6 & 35.1 & 18.9 & 24.3 & \\
\hline Reconstituida & 20.0 & 20.0 & 40.0 & 20.0 & \\
\hline Diagnóstico & & & & & \\
\hline MMC abierta & 25.5 & 21.6 & 27.4 & 25.5 & 0.85 \\
\hline MMC cerrada & 20.0 & 40.0 & 13.3 & 26.7 & \\
\hline $\begin{array}{l}\text { Espina bífida } \\
\text { oculta }\end{array}$ & 25.0 & 25.0 & 25.0 & 25.0 & \\
\hline $\begin{array}{l}\text { Ubicación de la } \\
\text { lesión }\end{array}$ & & & & & \\
\hline Lumbar & 14.8 & 22.2 & 25.9 & 37.0 & 0.39 \\
\hline Sacro & 23.5 & 17.6 & 35.3 & 23.5 & \\
\hline Lumbro-sacro & 26.7 & 40.0 & 20.0 & 13.3 & \\
\hline Dorso lumbar & 100 & - & - & - & \\
\hline Dorsal & 28.6 & 42.9 & 14.3 & 14.3 & \\
\hline Tórax & 50.0 & - & - & 50.0 & \\
\hline Ayuda para la marcha & & & & & \\
\hline $\begin{array}{l}\text { Camina sin } \\
\text { ayuda }\end{array}$ & 15.4 & 30.8 & 23.1 & 30.8 & 0.97 \\
\hline Soportes & 22.7 & 22.7 & 27.3 & 27.3 & \\
\hline $\begin{array}{l}\text { Uso de sillas de } \\
\text { ruedas }\end{array}$ & 28.6 & 25.7 & 22.9 & 22.9 & \\
\hline
\end{tabular}


de vida (OR: 0.96). En cuanto a las estrategias de afrontamiento el buscar diversiones relajantes (OR: 1.28) y fijarse en lo positivo (OR: 1.27) aumentan los niveles de la calidad de vida. Por otro lado, la falta de afrontamiento (OR: 0.75), auto inculparse y reservarlo para sí (OR: 0.81) se asocian a una disminución en los niveles de la calidad de vida.

Tabla 3. Fuerza de asociación entre los estilos y estrategias de afrontamiento con la calidad de vida

\begin{tabular}{lrrr}
\hline & \multicolumn{3}{c}{ Calidad de vida } \\
& OR & IC95\% & p \\
\hline Estilos de afrontamiento & & & \\
$\quad$ Resolver el problema & 1.06 & $1.03-1.09$ & 0.00 \\
$\quad$ Afrontamiento no productivo & 0.96 & $0.93-0.99$ & 0.00 \\
$\quad$ Referido a otros & 1.03 & $1.00-1.07$ & 0.03 \\
Estrategias de afrontamiento & & & \\
$\quad$ Concentrarse en resolver el & & & \\
problema & 1.22 & $1.11-1.36$ & 0.00 \\
$\quad$ Esforzarse y tener éxito & 1.17 & $1.03-1.34$ & 0.01 \\
$\quad$ Comprometerse con amigos & & & \\
íntimos & 1.15 & $1.06-1.26$ & 0.00 \\
Buscar pertenencia & 1.17 & $1.05-1.29$ & 0.00 \\
Fijarse en lo positivo & 1.27 & $1.12-1.44$ & 0.00 \\
Buscar diversiones relajantes & 1.28 & $1.04-1.57$ & 0.02 \\
$\quad$ Distracción física & 1.13 & $0.99-1.27$ & 0.05 \\
Preocuparse por el futuro & 1.13 & $1.02-1.25$ & 0.01 \\
$\quad$ Hacerse ilusiones & 1.07 & $0.95-1.21$ & 0.26 \\
Falta de afrontamiento & 0.75 & $0.66-0.87$ & 0.00 \\
Reducción de la tensión & 0.85 & $0.75-0.95$ & 0.00 \\
Ignorar el problema & 0.85 & $0.73-0.98$ & 0.02 \\
Auto inculparse & 0.81 & $0.72-0.91$ & 0.00 \\
Reservarlo para si & 0.81 & $0.72-0.93$ & 0.00 \\
Buscar apoyo social & 1.11 & $1.00-1.22$ & 0.03 \\
Acción social & 1.03 & $0.93-1.15$ & 0.55 \\
Buscar apoyo espiritual & 1.12 & $1.00-1.25$ & 0.05 \\
Buscar apoyo profesional & 1.08 & $0.99-1.19$ & 0.07 \\
Edad & 0.83 & $0.64-1.08$ & 0.16 \\
Sexo & & & \\
Hombre & 1.00 & - & - \\
$\quad$ Mujer & 0.52 & $0.22-1.22$ & 0.13 \\
Grado de instrucción & & & \\
$\quad$ Primaria & 1.00 & - & - \\
Secundaria & 1.21 & $0.34-4.33$ & 0.76 \\
Superior & 4.25 & $0.24-74.5$ & 0.32 \\
\hline
\end{tabular}

Tabla 3. continúa

\begin{tabular}{lccc}
\hline & \multicolumn{3}{c}{ Calidad de vida } \\
& OR & IC95\% & p \\
\hline Tipo de familia & & & \\
$\quad$ Nuclear & 1.00 & - & - \\
Monoparental & 0.70 & $0.11-4.27$ & 0.69 \\
$\quad$ Extensa & 0.78 & $0.30-2.01$ & 0.61 \\
Reconstituida & 0.97 & $0.18-5.20$ & 0.97 \\
Diagnóstico & & & \\
MMC abierta & 1.00 & - & - \\
MMC cerrada & 0.90 & $0.33-2.51$ & 0.85 \\
$\quad$ Espina bífida oculta & 0.95 & $0.15-5.89$ & 0.95 \\
Ubicación de la lesión & & & \\
Lumbar & 1.00 & - & - \\
$\quad$ Sacro & 0.64 & $0.21-1.91$ & 0.42 \\
Lumbro-sacro & 0.34 & $0.10-1.07$ & 0.06 \\
$\quad$ Dorso lumbar & - & - & - \\
$\quad$ Dorsal & 0.31 & $0.07-1.37$ & 0.12 \\
$\quad$ Tórax & 0.54 & $0.02-15.5$ & 0.71 \\
Ayuda para la marcha & & & \\
$\quad$ Camina sin ayuda & 1.00 & - & - \\
$\quad$ Soportes & 0.86 & $0.25-2.89$ & 0.80 \\
Uso de sillas de ruedas & 0.63 & $0.20-1.95$ & 0.42 \\
\hline
\end{tabular}

\section{DISCUSIÓN}

La investigación analizó la asociación entre el afrontamiento y la calidad de vida en adolescentes con EB. Los resultados obtenidos evidenciaron la relación positiva y estadísticamente significativa entre el afrontamiento y la calidad de vida. Asimismo, se relacionan significativamente los estilos productivos de afrontamiento resolver el problema y referencia a otros con niveles altos de la calidad de vida en los adolescentes con EB.

Se observó que entre los adolescentes con EB aumenta la calidad de vida cuando emplean los estilos de afrontamiento conocidos como resolver el problema y referencia a otros. Esto se explica debido a que los adolescentes que tienden a utilizar de manera activa sus recursos personales para hacer frente a sus vicisitudes, comparten sus preocupaciones, buscan soporte en su entorno social, familiar, espiritual y profesional podrían desarrollar estrategias que la permiten manejar, 
minimizar o tolerar las demandas de su condición de vida. Es decir, realizan el esfuerzo cognitivo y conductual para regular las perturbaciones que afectan negativamente su salud (16).

La calidad de vida se incrementa con las estrategias de afrontamiento como buscar diversiones relajantes y fijarse en lo positivo, otros estudios reportan resultados similares (17). El adolescente con EB que tiene actividades de ocio, como por ejemplo leer libros, tocar un instrumento musical, entre otros, mejora su bienestar emocional y social (18). Asimismo, cuando se inclina a ser optimista o presta más atención a los aspectos positivos de las situaciones presentes y confía en el entorno social, esto genera mayor cantidad de soluciones a sus problemas o vicisitudes. De consecuencia contribuyen a una adecuada percepción de su calidad de vida (17).

En cambio los niveles de calidad de vida disminuyen con un estilo de afrontamiento no productivo. Se observó que los adolescentes con EB se autoinculpan por su condición o se preocupan excesivamente ante sus dificultades y su futuro. Incluso, disponen de una disminuida capacidad para resolver problemas, manifiestando actitudes evasivas y tienen una visión negativa de la vida. Por ende, estos estilos favorecen las perturbaciones que afectan la salud ya que se orientan a no abordar sus dificultades (19). Asimismo, se limitan en la búsqueda de su autonomía e independencia afectando negativamente su calidad de vida (20).

Del mismo modo, la calidad de vida disminuye cuando el adolescente con EB muestra estrategias no productivas como: la falta de afrontamiento, el autoinculparse y el reservarlo para sí. Otro estudio reporta resultados similares (21). Como resultado de ello, los adolescentes con EB asumen los siguientes procesos ante sus vicisitudes: limitan sus capacidades para enfrentar sus dificultades. Sus conductas indican que se responsabiliza de sus problemas y evitan que su entorno conozca sus sentimientos ante esta situación problemática. En consecuencia no logran dominar o tolerar sus demandas con esos estilos de vida, puesto que no les permiten encontrar soluciones, evitan todo aspecto positivo de su situación, aumentando su malestar emocional y tienden a desarrollar síntomas psicosomáticos. Todo ello influye en la disminución de su calidad de vida (19).
Entre las limitaciones del estudio se encuentra el sesgo de cortesía, es decir, que algunos evaluados para complacer al entrevistador ofrecen respuestas satisfactorias. Además, no se evaluaron factores fisiológicos que pudieran incidir en la calidad en la percepción de la calidad de vida en este sentido los resultados deberán ser contrastados con otros estudios debido a la incipiente indagación en el contexto nacional.

\section{CONCLUSIONES}

Los niveles altos de la calidad de vida se asocian fuertemente con el estilo resolver el problema y la estrategia de buscar diversiones relajantes. Sin embargo, una disminución de la calidad de vida se asocia con el estilo afrontamiento no productivo $\mathrm{y}$ las estrategias de falta de afrontamiento, auto inculparse y reservarlo para sí. Por ello, es necesario crear programas de intervención para reducir las secuelas de estilos y estrategias de afrontamiento no productivos en los adolescentes con EB.

Teniendo en cuenta estos resultados es necesario crear programas de intervención que incluyan opciones en la educación, las actividades de ocio, los juegos recreativos, entre otros factores que permiten el desenvolvimiento del adolescente con EB con la finalidad de reducir las secuelas de estilos y estrategias afrontamiento no productivos. Dada la amplia gama de complejos desafíos de salud y de rehabilitación en estos adolescentes son necesarios mayores estudios que consideren más factores relacionados a su condición de vida. Nuestra comprensión del fenómeno en personas con EB se puede mejorar en la medida que se elimine el vacío de conocimiento sobre el tema.

\section{REFERENCIAS BIBLIOGRÁFICAS}

1. Peterson VO, Gutiérrez MB. Atención del niño con mielomeningocele [tesis]. Ciudad de México: Instituto Nacional de Pediatría; 1990.

2. De la Rosa G, Domínguez L. Epidemiología, diagnóstico y tratamiento del mielomeningocele en el Hospital Infantil Napoleón Franco Pareja de Cartagena [tesis doctoral]. Cartagena de Indias: UNIVERSIDAD DE CARTAGENA; 2011.

3. Salas AM, León BC, Jiménez GA, Ramírez JR. (2001). Epidemiología del mielomeningocele en niños menores de un año de edad en el Instituto Nacional de Pediatría. Revista mexicana de Medicina física y rehabilitación. 2001;13(2):50-54. 
4. De la Rosa G, Domínguez L. Epidemiología, diagnóstico y tratamiento del mielomeningocele en el Hospital Infantil Napoleón Franco Pareja de Cartagena [tesis doctoral]. Cartagena de Indias: UNIVERSIDAD DE CARTAGENA; 2011.

5. Salas AM, León BC, Jiménez GA, Ramírez JR. (2001). Epidemiología del mielomeningocele en niños menores de un año de edad en el Instituto Nacional de Pediatría. Revista mexicana de Medicina física y rehabilitación. 2001;13(2):50-54.

6. Sanabria H, Tarqui-Mamani C, Arias J, Lam $\mathrm{N}$. Impacto de la fortificación de la harina de trigo con ácido fólico en los defectos del tubo neural, en Lima, Perú. An Fac med. 2013; 74(3):175-180.

7. Blum RW, Pfaffinger K. Myelodysplasia in childhood and adolescence. Pediatrics in review/American Academy of Pediatrics. 1994;15(12):480-484.

8. Wolman C, Basco DE. Factors influencing self-esteem and self-consciousness in adolescents with spina bifida. Journal of adolescent Health. 1994;15(7):543548-545.

9. Frydenberg E, Lewis R. Coping with Stresses and Concerns during Adolescence: A Longitudinal Study. 1997.

10. Botero P. Discapacidad y estilos de afrontamiento: una revisión teórica I. Revista Vanguardia Psicológica Clínica Teórica y Práctica. 2013;3(2):196-214.

11. Dickens P, McMillen JS. Growing Up with Spina Bifida: what we have learned. The North Carolina Office on Disability and Health (NCODH). 2000.

12. Frydenberg, E. Adolescent coping: Theoretical and research perspectives. Londres: Psychology Press; 1997.

13. The WHOQOL Group. WHOQOL: position paper from the World Health Organization. Social Science and Medicine. 1995;41(10):1403-1409.

14. Olson DH, Barnes HL. Family inventory of quality of life [tesis]. Minneapolis, MN: University of Minnesota; 1982.

15. Engel GL. From biomedical to biopsychosocial: Being scientific in the human domain. Psychosomatics. 1997;38(6):521-528.

16. Frydenberg, E. The coping strategies used by capable adolescents. Australian journal of guidance \& counselling. 1993;3(1):15-23. https://www.cambridge.org/core/journals/journ al-of-psychologists-and-counsellors-in-
schools/volume/BD95E43E3DEAF1C4544F4 A714DB73A2C

17. Lazarus RS, Folkman S. Transactional theory and research on emotions and coping. European Journal of personality. 1987;1(3):141-169.

18. Botero PA, Londoño C. Factores psicosociales predictores de la calidad de vida en personas en situación de discapacidad física. Acta Colombiana de Psicología. 2013;16(2):125137

19. Pollard C, Kennedy P. A longitudinal analysis of emotional impact, coping strategies and post-traumatic psychological growth following spinal cord injury: A 10-year review. British journal of health psychology. 2007;12(3):347362.

20. Gaviria AM, Vinaccia S, Riveros MF, Quiceno JM. Calidad de vida relacionada con la salud, afrontamiento del estrés y emociones negativas en pacientes con cáncer en tratamiento quimioterapéutico. Psicología desde el Caribe. 2007;(20):50-75. Disponible en:

http://www.redalyc.org/articulo.oa?id=213020 04

21. Contreras F, Esguerra GA, Espinosa JC, Gómez V. Estilos de afrontamiento y calidad de vida en pacientes con insuficiencia renal crónica (IRC) en tratamiento de hemodiálisis. Acta Colombiana de Psicología. 2007;10(2):169-179. Disponible en: http://www.redalyc.org/articulo.oa?id=798102 16

22. Domínguez L. Contextualización de la espina bífida en Cádiz: análisis y propuesta de intervención. Un enfoque familiar [tesis]. Cádiz: Universidad de Cádiz; 2015.

23. Pucheu S, Consoli SM, D'Auzac C, Français P, Issad B. Do health causal attributions and coping strategies act as moderators of quality of life in peritoneal dialysis patients? Journal of psychosomatic Research. 2004;56(3):317322. 
ISSN 2519-0652 CASUS.2017;2(3):139-146

\section{Understanding coping and quality of life in adolescents with spina bifida}

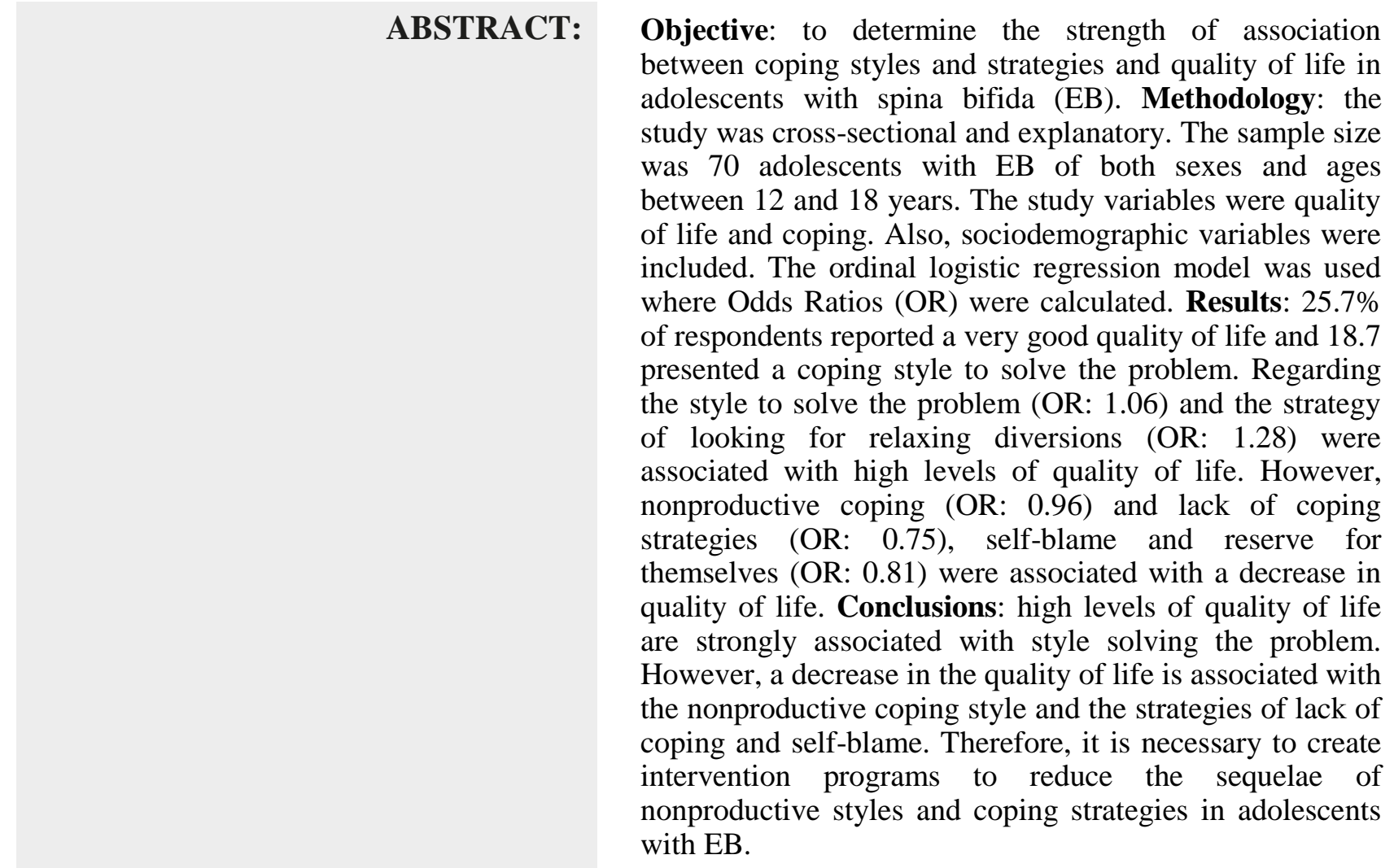

KEY WORDS: Coping; Quality of life; Spina bifida. 